\title{
On the feasibility of inversion methods based on models of urban sky glow
}

\author{
Z. Kolláth ${ }^{\mathrm{a}, \mathrm{b}, *}$, B. Kránicz ${ }^{\mathrm{c}}$ \\ ${ }^{a}$ University of West Hungary, Savaria Campus, Szombathely, Hungary \\ ${ }^{b}$ MTA CSFK Konkoly Observatory, Budapest, Hungary \\ ${ }^{c}$ University of Pannonia, Veszprém, Hungary
}

\begin{abstract}
Multi-wavelength imaging luminance photometry of sky glow provides a huge amount of information on light pollution. However, the understanding of the measured data involves the combination of different processes and data of radiation transfer, atmospheric physics and atmospheric constitution. State of the art numerical radiation transfer models provide the possibility to define an inverse problem to obtain information on the emission intensity distribution of a city and perhaps the physical properties of the atmosphere. We provide numerical tests on the solvability and feasibility of such procedures.
\end{abstract}

Keywords: Light pollution, Radiative transfer, Light scattering

\section{Introduction}

The Hungarian protected area network (national parks, wildlife reserves) almost overlaps with the dark sky areas. This fact indicates their mission in protecting dark skies, as nature conservation is deeply interrelated with protecting the nocturnal landscape. Our goal was to identify those areas, which could be suitable for the nomination to be dark sky parks. In Europe it is hard to find really dark places. Even far from large cities and local settlements there is an increased level of sky brightness due to excessive sources hundreds of kilometres away from that location. Therefore night sky quality monitoring becomes an important part of nature conservation. We performed imaging sky luminance measurements at different locations in the country as part of the designation of natural park areas for International Darks Sky Parks (IDSP) recognized by the International Dark Sky Association (IDA). As a result of our work the Zselic Landscape Protection Area and the Hortobágy National Park were recognized as IDSP in 2009 and 2011.

A possible method to qualify the light pollution (sky glow) over a large area is to make maps of night sky brightness in and around a protected area. A possible way to generate such maps is to measure the (average) luminance of a portion of the night sky by a luminance meter (e.g. Sky Quality Meter). If such measurements are made on a dense enough geographic grid, the sky glow of the territory can be mapped. However, significantly more information can be gathered by imaging photometry of the sky. In addition, recent techniques to survey light pollution (e.g. [1] and [2]) provide the spatial distribution

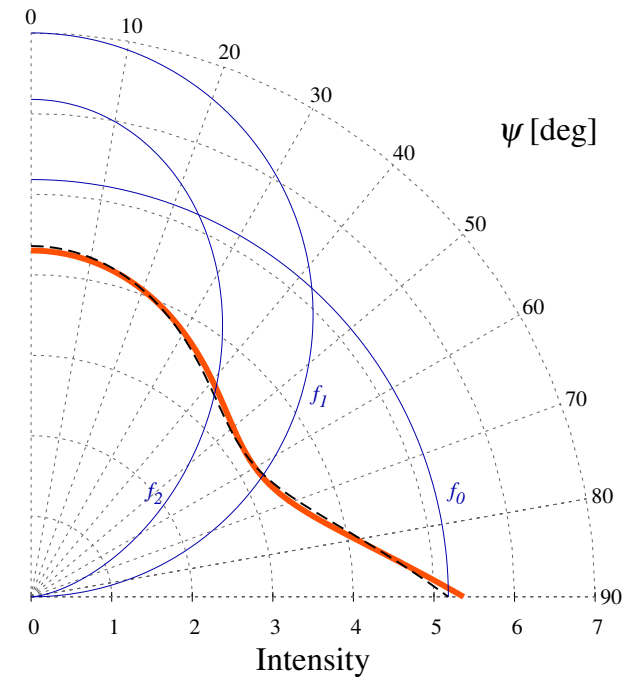

Figure 1: Garstang's upward angular intensity distribution function (thick solid line). The fit with the first three $F_{l}$ polynomials is shown by thin solid curve. The weighted polynomials $f_{k}=c_{k} F_{k}$, constrained by $f_{1}-f_{2}+f_{3}=$ fit, are displayed by thin dashed curves. The intensity scale is arbitrary (see the text for more details).

\footnotetext{
* Corresponding author

Email address: kollath@konkoly.hu (Z. Kolláth)
} 
of artificial sky luminance as a function of different parameters (wavelength, sky position, etc.). Recent Digital Single Lens Reflex (DSLR) cameras provide a new opportunity to monitor the quality of the night sky and light pollution. Cameras that are able to save images in an unaltered raw format, can be calibrated to get measurements of the luminance of the sky on a physical scale. Then the photo of the night sky can be converted to false colour images, which represents the distribution of sky brightness [2]. Such calibrated images of the light domes over cities provides enough information to interpolate or even extrapolate them to a larger area if the measurements are combined with numerical radiation transfer modelling.

The general procedure of light pollution modelling uses the spatial distribution and the characteristic properties of light sources and the physical parameters of the atmosphere as an input for the calculations, and then the distribution of the luminance of sky glow is provided as the result. The basic elements of these methods can be found e.g. in [3] and [4] and references therein. The output of the models then can be compared with measurements to verify the physical ingredients and input parameters of the numerical calculations. These models are based on numerical integration of the radiation transfer equations. Another possible way is to perform Monte-Carlo simulation of photon packets (see e.g. [5]). For the tests in this paper we used the second method.

The combination of models and observations gives the possibility to invert the procedure and to use light pollution models to predict the quantities of the emission sources from observations. Details of this inversion method are provided in the paper.

\section{Models}

To interpret the sky brightness measurements and to provide models of the light pollution, we have developed a Monte-Carlo radiation transfer code. In clear air (no clouds), the propagation of light is determined by Rayleigh scattering for molecules and by Mie scattering for aerosols. For most of the calculations of this paper, absorption was neglected for simplicity. The mean free path of photons in terrestrial atmosphere is several tens of kilometres, depending on the aerosol content and elevation. It gives a natural choice to use Monte-Carlo simulation of the light propagation since the observed photometric quantities are statistical averages of photon packets. For detailed description of Monte-Carlo radiation transfer calculations see e.g. [5] Here we summarize only the major ingredients of the code: Mie scattering is approximated by the HenyeyGreenstein phase function (see e.g. in [5]). This makes some approximation in our calculations, but the use of different phase functions does not alter our main conclusions. Multiple scattering is calculated, the code follows the photon packets until they leave the atmosphere at the outer boundary or absorbed on the ground. Both forward and reverse (the photon packets are initiated at the detector)
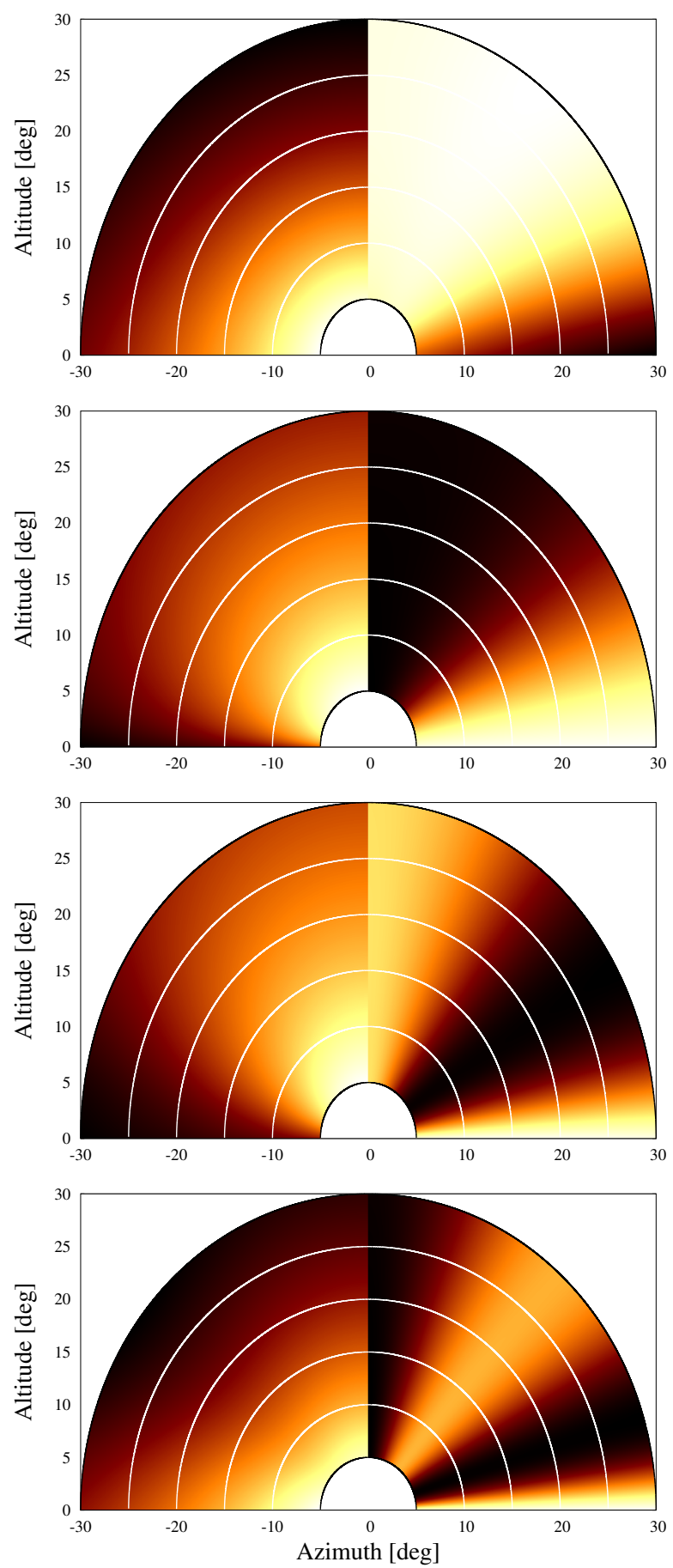

Figure 2: The calculated luminance for $l=0,1,2,3$ (from top to bottom). The left side of each figure displays the real luminance distribution $\left(\Xi_{l}\right)$ on the sky, while the right quadrants show the elementary distributions based on the matrix $\mathbf{H}$ resulted in singular value decomposition. The half circles indicate the $\rho$ values of the grid, which is used to interpolate the intermediate values. The fixed parameters are: $R=10 \mathrm{~km}, H_{a}=1.5 \mathrm{~km}, \tau_{a}=0.1$. 
calculation is possible. The efficiency of the Monte-Carlo simulation is greatly increased by the peel-off technique [6].

The spatial distribution of sky glow is determined by spatial distribution of the total flux radiated to the atmosphere and the upward-directed angular luminous (or radiant) intensity distribution $I(\psi)$ of the light polluting sources. Please note that in this paper we simply use intensity to denote luminous intensity. But in most of the circumstances the results are valid for radiant quantities as well. In all the tests we calculated radiation transfer for a set of wavelengths (between $380 \mathrm{~nm}$ and $780 \mathrm{~nm}$ in $5 \mathrm{~nm}$ steps) and integrated the monochromatic results with CIE 1931 photopic visibility function $(V(\lambda))[7]$ to get the luminous quantities. We note that the calculations can be performed with the scotopic visibility function $\left(V^{\prime}(\lambda)\right)$ as well. In our study the intensity distribution is approximated by a series of spherical harmonics. We concentrate on the axial symmetric distribution, then the series is reduced to the associated Legendre polynomials $P_{l m}(\cos \psi)$ with $m=0$. To avoid negative intensities in the calculations, the elementary intensity distributions are slightly modified: $F_{0}=P_{00}, F_{1}=P_{10}, F_{2}=P_{20}+\frac{1}{2} P_{00}$, $F_{3}=P_{30}+\frac{1}{2} P_{00}, F_{4}=P_{40}+\frac{1}{2} P_{00}$.

To demonstrate the effectiveness of this series, the fit of the generally used Garstang's distribution [8] is displayed in Figure 1. The conclusions of this paper do not depend on the intensity scale. However, for this figure and for all the other polar diagrams in the paper we used an arbitrary scale that gives the luminous intensity assuming that the total luminous flux of the source is 100 units (e.g a flux of 100 lumens gives the intensity in candelas). Garstang's standard upward intensity distribution is a sum of the Lambertian reflection (identical to $F_{1}=P_{10}(\cos \psi)$ ) and a distribution defined by the fourth power of the zenith angle $\left(\psi^{4}\right)$. A fit with the first three polynomials $\left(F_{0}, F_{1}\right.$, $F_{2}$ ) gives a sufficient result. A 5 th order fit (used for the tests discussed in this paper) is almost undistinguishable from the original upward angular light distribution.

\subsection{Model components}

A grid of models with different atmospheric conditions is calculated by our Monte-Carlo radiation transfer code. To test the effect of humidity and aerosol content in the atmosphere, a two parameter model of aerosol distribution is used. The aerosol optical thickness at 500nm $\left(\tau_{a}\right)$ is varied from 0 to 0.4 (for a fixed grid of $\tau_{a}=0.0,0.02$, $0.05,0.10,0.20,0.30,0.40)$, and calculations for three different values of the aerosol scale height $H_{a}=1.2,1.5$ and $2 \mathrm{~km}$ were performed. This range of parameters provides results for horizontal visibility in the range of $\approx 10-200 \mathrm{~km}$. For each $\tau_{a}, H_{a}$ combinations the propagation of photon packets was calculated for all visible wavelengths with an increment of $5 \mathrm{~nm}$ and $F_{j}$ elementary upward angular light distributions. The calculations provide the distribution of the artificial radiance of the sky as a function of the distance from the source $(R)$, the sky coordinates (azimuth,

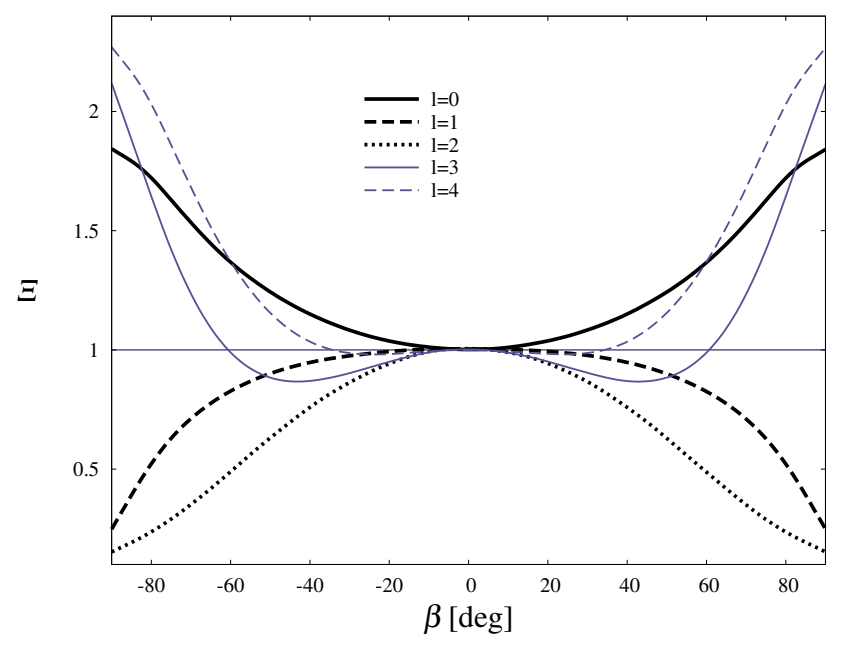

Figure 3: The calculated luminance $\left(\Xi_{l}\right)$ for the fixed $\rho=20^{\circ}$, the other parameters are the same as the ones indicated in Figure 2. For better visibility all the curves are normalised at beta $=0$ to give $\Xi_{l}(0) \equiv 0$.

elevation) and wavelength $(\lambda)$. The resulting database still contains redundant information if the luminance distribution is calculated for the whole upper hemisphere. The sky glow over a city is well represented by the luminance distribution as a function of azimuth and elevation, that is the standard image of the sky over the light sources. However, a coordinate transformation greatly helps in the fitting procedure. Instead of azimuth and elevation we use two other parameters, $\rho$ and $\beta$, defined by azimuth $=\rho \sin (\beta)$ and elevation $=\rho \cos (\beta)$. The azimuth is measured from the direction of the city centre. Moreover, instead of the whole spatial distribution on the sky (e.g. $L(\rho, \beta)$ ), to reduce the database, the radiance data were calculated only for limited values of $\rho=5,10,15,20,25$ and 30 degrees. Data for a fixed $\rho$ represent the radiance and finally the luminance along specific (circular) curves with fixed angular distance $\rho$ from the direction of the source on the sky. According to our experience the luminance along these curves provides the information to interpolate the distribution for the whole $5^{\circ}<\rho<30^{\circ}$. The limits of the $\rho$ range can be increased, but for the recent test this interval turned out to be sufficient. The resulting model data can be summarized as $X_{l}\left(\tau_{a}, H_{a}, R, \rho, \beta, \lambda\right)$, where the grid for all the independent variables is defined so that it is possible to interpolate for the whole parameter space.

The spectral distribution of the sources is expected to be a combination of the spectra of a sodium lamp and a white LED. However the general results do not really depend on the spectral distribution. Then the numerical results are reduced to Luminance by integrating the calculated distributions with the assumed source spectral distribution $S(\lambda)$ and the $V(\lambda)$ visibility function:

$$
\Xi_{l}\left(\tau_{a}, H_{a}, R, \rho, \beta\right)=\int X_{l}\left(\tau_{a}, H_{a}, R, \rho, \beta, \lambda\right) S(\lambda) V(\lambda) d \lambda
$$



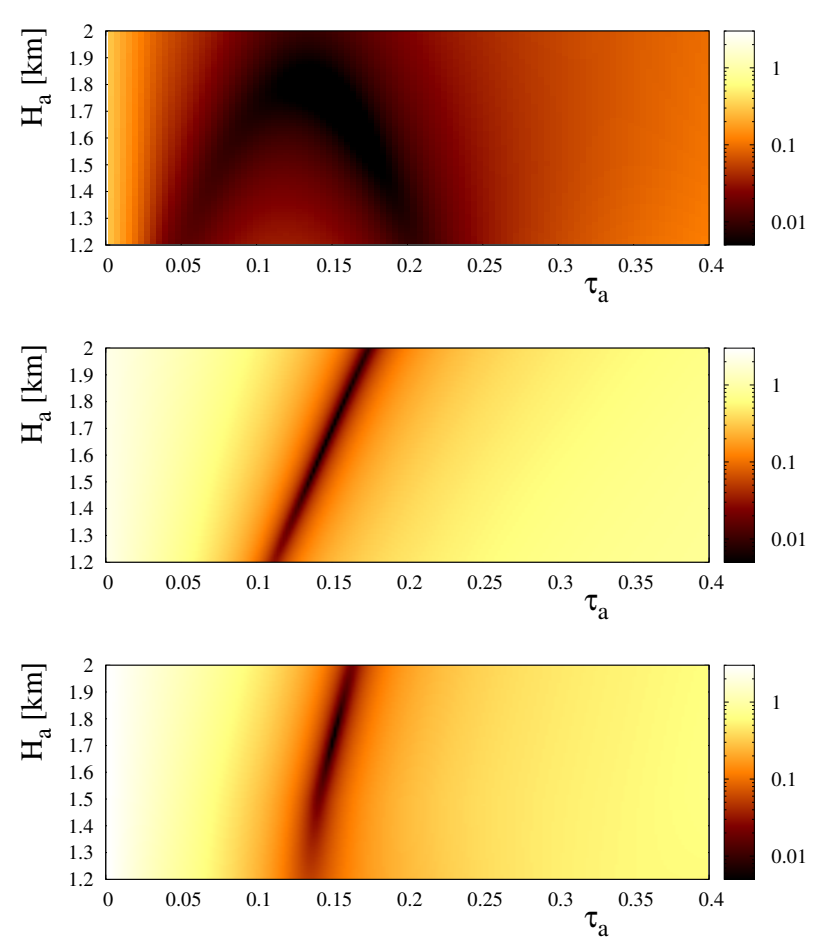

Figure 4: Prediction error (in log scale) obtained by fitting the $\rho=$ $20^{\circ}$ curve at $R=10 \mathrm{~km}$. Top: for the $\rho=10^{\circ}$ curve at $R=10 \mathrm{~km}$; Middle: for the $\rho=20^{\circ}$ curve at $R=20 \mathrm{~km}$; and Bottom: for fixed $\rho=20^{\circ}$ and $\beta=0^{\circ}$ in the range $R=20-60 \mathrm{~km}$.

\subsection{Model results}

Figure 2 displays the model results $(\Xi)$ for four upwarddirected angular distribution base functions $F_{0}, F_{1}, F_{2}$ and $F_{3}$ and fixed values of $R=10 \mathrm{~km}, H_{a}=1.5 \mathrm{~km}, \tau_{a}=0.1$. The white and black circular curves indicate where the actual $\Xi$ luminances are calculated. The images are obtained by a 2 dimensional cubic spline interpolation on the $\beta$ - $\rho$ plane. The $\Xi(\rho, \beta)$ curves vary quite smoothly when $\rho$ is changed, and the $\Delta \rho=5^{\circ}$ sampling turned out to be sufficient. To display the model results for all the used base functions $F_{l}$, the results for fixed $\rho=20^{\circ}$ are shown in Figure 3.

\section{Inversion}

The $\Xi_{l}\left(\tau_{a}, H_{a}, R, \rho, \beta\right)$ model results on the discrete grid provide the directional and spatial distribution of artificial sky luminance for a spectral distribution, atmospheric condition and a base function $F_{j}$ which is a member of a series representing the directional distribution of the intensity of the emission of a given light source. For a fixed set of $\tau_{a}, H_{a}, R, \rho$, the models can be represented by the matrix $\mathbf{M}$ given by $M_{l k}=\Xi_{l}\left(\tau_{a}, H_{a}, R, \rho, \beta_{k}\right)$; where $\beta_{k}$ represents the grid values of the angular parameter $\beta$.

The simplest observations are given by the measurements of the Luminance along a fixed curve with a given $\rho$. These observations are denoted by $L_{k}(\rho)=L\left(\rho, \beta_{k}\right)$. We note, that it is straightforward to measure $L_{k}$ from imaging photometry / luminance measurements since the $\rho$ and $\beta$ angles can be determined by astrometry of the bright stars in the images.

Assuming that the measurement is taken at a given distance $R$ from the source, then both $R$ and $\rho$ are fixed. The only undetermined parameters are $\tau_{a}$ and $H_{a}$. For a given pair of $\tau_{a}$ and $H_{a}$ the observations are fitted by the following sum:

$$
\hat{L}_{k}=\sum_{l=0}^{4} c_{l} M_{l k}
$$

To fit a sequence of observations sufficiently, the Singular Value Decomposition (SVD) of $\mathbf{M}$ was calculated to obtain the representation: $\mathbf{M}=\mathbf{U S V}^{*}$. The resulting left orthogonal vectors of $\mathbf{U}$ then can be used as new basis vectors, whose coefficients are simply given by scalar products of vectors, and then the coefficients of the starting $F_{l}$ base functions can be calculated by a simple transformation. Finally the model calculations were transformed to the matrix defined by $\mathbf{H}=\mathbf{V S}^{-1} \mathbf{U}^{*}$. Then, for a fixed set of parameters of the atmospheric model, the coefficients in Eq. 2. can be calculated by $\mathbf{c}=\mathbf{H} * \mathbf{L}$. All the model calculations are then represented by $\mathbf{H}$ as a function of $\mathbf{H}\left(\tau_{a}, H_{a}, R, \rho\right)$ and the coefficients are given as a single matrix by vector multiplication. Then, at least in principle, the inversion can be performed by simple arithmetic.

The obtained $\mathbf{H}\left(\tau_{a}, H_{a}, R, \rho\right)$ system provides a fast way to perform an inverse problem calculation based on light pollution measurements. Although the calculation of the necessary grid of models is numerically very intensive and time consuming, after the initial model grid calculation has been performed, the interpretation of the measurement is simplified by the use of the method. In this paper we fixed $\rho=20^{\circ}$ and $R=10 \mathrm{~km}$ for the primary observations. We generated synthetic observational data with the Monte-Carlo radiative transfer code. Our tests have shown, that for fixed $\rho$ and $R$ values any possible smooth synthetic observations can be fitted successfully independently of the atmospheric parameters in the models. It means that such a simple observation cannot give any estimate on the atmospheric conditions. The fitted $c_{l}$ coefficients depend on $\tau_{a} H_{a}$ and $R$. No definite values can be obtained.

To give an estimate for these values, we have to add additional observations to constrain the system. The fitted $c_{l}\left(\tau_{a}, H_{a}\right)$ coefficients to the base observations at $\rho=20^{\circ}$ and $R=10 \mathrm{~km}$, however, can be used to predict the observations for other positions on the sky and other geometric locations based on the full set of $\mathbf{M}$. The error of the prediction can be defined as

$$
\sigma^{2}\left(\tau_{a}, H_{a}\right)=\left\langle(\hat{L}-L)^{2}\right\rangle
$$

where the expected value is calculated for different sets of observations. To demonstrate the effects on different observations, we constructed a set of synthetic observations with $\tau_{a}=0.15$ and $H_{a}=1.7$. We calculated the error of the predictions for three different sets of observations: (i) at the same location at $R=10 \mathrm{~km}$ but with 


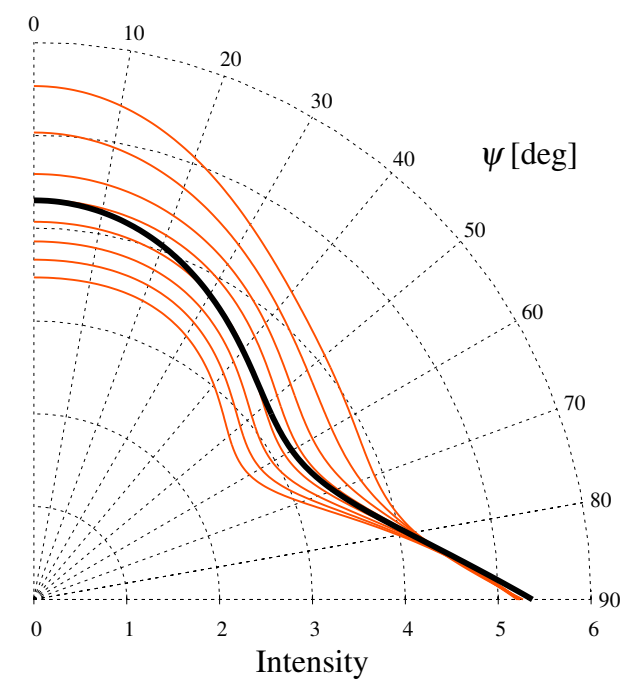

Figure 5: The initial upward angular intensity distribution function (thick solid line), and its best predictions at different $H_{a}=1.3,1.4$, $1.5,1.6,1.7,1.8,1.9$ and $2.0 \mathrm{~km}$ (from top to bottom). The intensity scale is arbitrary.

$\rho=10^{\circ}$; (ii) at a different location of $R=20 \mathrm{~km}$, with $\rho=20^{\circ}$; and (iii) with fixed $\beta=0$ and $\rho=20^{\circ}$ but with different distances from the source $(10 \mathrm{~km}<R<50 \mathrm{~km})$. The resulting prediction errors are presented in Figure 4. From these tests it can be concluded that observations from a single site cannot give a good choice for inversion. The observed curves for different $\rho$ values are correlated, the atmospheric parameters can be calculated only with a high error. On the contrary, observations only at a fixed $\rho$ but at two different locations provide a narrow range on the $\tau_{a}, H_{a}$ plane, especially for $\tau_{a}$. However, the aerosol scale height is still undetermined, but a single line connecting the two parameters can be determined. By combining the two upper figures the correct input data can be well estimated. The third test case, when observations are performed at many different locations, does not improve significantly the determination of atmospheric parameters, the lower two plots are highly correlated, especially if noise and other error sources are taken into consideration. We have to note, that this finding also gives the possibility to predict the sky luminance for different distances, based on measurements only at two different locations.

To test the possible errors on inversion methods of the sources, we selected a set of $\tau_{a}, H_{a}$ pairs from the middle plot of Figure 4, which provide the best fits for the observations. For each combinations of the parameters, we reconstructed the upward intensity distribution. The results are displayed in Figure 5. The near horizon distribution is predicted well independently of the scale height, but for the whole range of $\psi$ only the curves close to the correct $H_{a}$ and $\tau_{a}$ give satisfactory agreements.

\subsection{The effect of extended sources}

In the above discussion it was assumed that the source had a negligible horizontal dimension. In real situations,
0 10 20

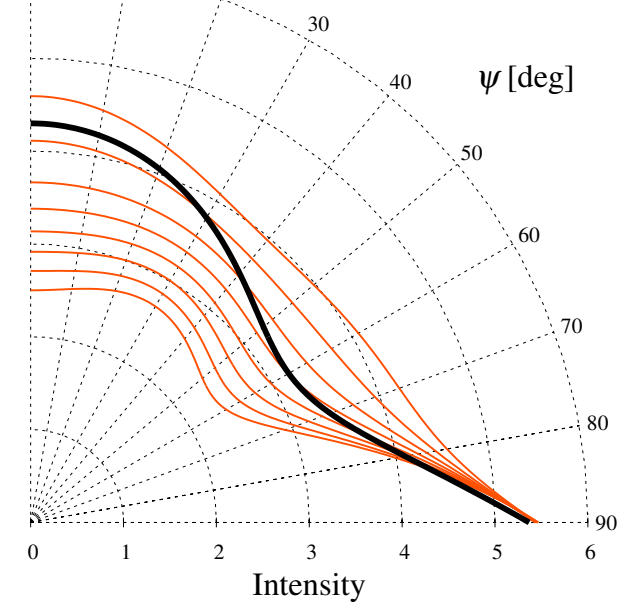

Figure 6: The initial upward angular distribution function (thick solid line), and its best predictions at different $H_{a}=1.3,1.4,1.5$, 1.6, $1.7,1.8,1.9$ and $2.0 \mathrm{~km}$ (from top to bottom). The synthetic observations are generated by an extended source, and it is fitted by a point source. The intensity scale is arbitrary.

the spatial distribution of the sources should be taken into consideration. The use of extended sources is straightforward, since the model grid can be interpolated to each source and the linear combination of the resulted sky glow distributions gives the model of night sky darkness. This method, however, is not suitable for inversion. Instead, the spatial distribution of the sources can be fixed, and a uniform effective upward-directed angular light distribution can be fitted to the observations. This method can be used to estimate the whole light output and its distribution of a settlement.

To estimate the possible errors due to extended sources, we calculated the synthetic observations for an extended source given by point sources within a circle of $0.5 \mathrm{~km}$ radius and fitted these data by models of point sources. A main conclusion of this test is that the best solutions estimate the aerosol optical thickness and scale height quite well, as can be seen by the comparison of Figures 4 and 7. The second plot differs from the previous one just in the construction of synthetic observations. Surprisingly, the best fit to the observations at the two distances and with $\rho=20^{\circ}$ gives a a relatively good overall fit as well. For example the fitted intensity distribution of the source is displayed in Figure 6. The inversion gives a satisfactory match, especially close to horizontal directions. Since the light output at low angles provides the major impact on sky quality, the inversion method provides a useful estimate for the light pollution sources.

If the size of the extended source increases, then the goodness of the fit degrades. The prediction error on the $H_{a}-\tau_{a}$ plane also increases and the 'valley' on the plot broadens, giving more uncertainty in the inversion. Then the model should incorporate an estimate of the source distribution. 


\subsection{Effect of aerosol scattering properties}

The exact form of the aerosol scattering phase function gives an additional uncertainty in the results. The majority of the tests discussed in this paper were performed with the one parameter Henyey-Greenstein phase function. It is a good first approximation of Mie scattering, but misses the correct level of backscattering and sometimes underestimates the forward scattering. In order to test the effect of the scattering phase-function, we calculated a set of models with a two term Henyey-Greenstein (TTHG) phase function ([9]):

$$
P_{T T H G}(\mu, \alpha, g, h)=\alpha P_{H G}(\mu, g)+(1-\alpha) P_{H G}(\mu,-h) ;
$$

where $P_{H G}$ is the standard Henyey-Greenstein phase function, and in addition to the usual asymmetry parameter $g$ a second one, denoted by $h$, is introduced to add an additional back scattering. We fixed $\alpha=0.5$ for the recent calculations. The asymmetry parameters were adjusted in the ranges of $g=0.4-0.95$ and $h=0.0-0.4$. The other atmospheric parameters were set to $H_{a}=1.7 \mathrm{~km}$ and $\tau_{a}=0.15$. The same method was followed as for the fit of the scale height and aerosol optical thickness, but now the parameters of the TTHG were fitted. The synthetic observations were created with $g=0.75 h=0.25$. The resulting prediction error is presented in Figure 8. Again, there is a set of parameter pairs $(g, h)$ which provides almost equally good results. Similarly to the previous sections, we tested the goodness of the fitted upward angular intensity distribution function. The results are displayed in Figure 9. This test clearly demonstrates that the exact form of the aerosol scattering phase function has only a limited effect on the fit of the source distribution function. We have got very similar results when the aerosol scattering albedo was taken into consideration (setting it to 0.9 uniformly for the whole wavelength range.) But we have to note that further tests are needed and we plan to extend our survey to a wider parameter range.

\subsection{Effect of single or multiple scattering}

Some of the published light pollution models apply single scattering, or limit the calculations to double scattering. In the Monte-Carlo calculations there are no restrictions for the number of scattering events. However, it is possible to finish the ray tracing of photon packets at a given scattering number. Then Monte-Carlo modelling gives a good tool to estimate the errors in inversion when single or double scattering codes are used.

For this test we created model grids by limiting the calculations to single, double or triple scattering. When the synthetic observation with $H_{a}=1.5 \mathrm{~km}, \tau_{a}=0.2$ values was fitted by the models with the same atmospheric parameters, but with different level of scattering, the resulted upward angular intensity distribution is deteriorated significantly. Figure 10 displays the difference of the fit from the original curve. Single scattering overestimates the emission at low angles and underestimates the intensity
Table 1: Mean error of the fit of the upward angular intensity distribution for different approximation in scattering number

\begin{tabular}{cccc}
\hline$\tau_{a}$ & $\sigma_{1}[\%]$ & $\sigma_{2}[\%]$ & $\sigma_{3}[\%]$ \\
\hline 0.02 & 4 & $<1$ & $<1$ \\
0.05 & 8 & 2 & 1 \\
0.10 & 20 & 4 & 2 \\
0.20 & 43 & 14 & 4 \\
0.30 & 65 & 27 & 11 \\
0.40 & 85 & 40 & 17 \\
\hline
\end{tabular}

at high elevations. The error of the fit strongly depends on the aerosol optical thickness. For fixed aerosol scale height $\left(H_{a}=1.5 \mathrm{~km}\right)$ we calculated the goodness of the fit as a function of $\tau_{a}$. The results are summarised in Table 1 , where the standard deviation of the fitted distribution from the real one is denoted by $\sigma_{N}$, where $\mathrm{N}$ gives the maximum number of possible scattering in the model. As expected, in clean air single scattering gives a good estimation in the inversion. However, for higher values of $\tau_{a}$ even double scattering provides false results. We have to note, that $\tau_{a}=0.3$ cannot be considered as a very high value, since in this model it is equivalent to a horizontal visibility of $18 \mathrm{~km}$. In addition, when the atmospheric parameters are also fitted, the best match of the observations is obtained at altered $\tau_{a}$ and $H_{a}$ pairs.

\subsection{Effect of colour}

The spectral composition of the sky glow can be determined by colour or spectral measurements of the sources. The colour of the artificial sky glow can also be estimated by calibrated DSLR photometry from the RGB channels of the camera. To approximate the possible error due to colour mismatch of the models and observations, we selected two extreme light sources: While the model was calculated for high pressure Sodium lighting, the synthetic observations were created by assuming cool white LED lighting. The choice of the Sodium lamp and the cool white LED is based on the fact that public lighting nowadays is experiencing certain changes towards the usage of white LEDs that have much higher colour rendering indices than that of the Na-lamp. All the other constituents are the same as those in the previous section. Even with this extreme difference, the error of the fit remains in the range of $3-5 \%$ independently of the aerosol optical thickness. Larger errors are expected only in extremely clean air, when the Rayleigh scattering dominates the radiation transfer. A simple measure of the colour of skyglow then can minimize the effect of colour to a level below the measurement's error.

\section{Summary}

We performed a test on the feasibility of urban skyglow inversion method by modelling the propagation of 


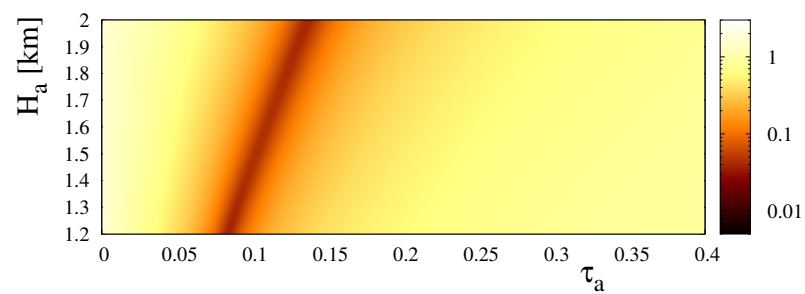

Figure 7: Same as the middle plot of Figure 4, but the synthetic observations were calculated with an extended source.

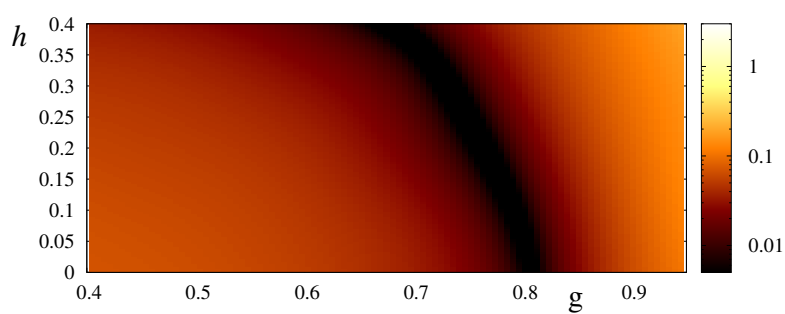

Figure 8: Prediction error (in log scale) as a function of the TTHG parameters, obtained by fitting the data in the same way as in the middle panel of Figure 4.

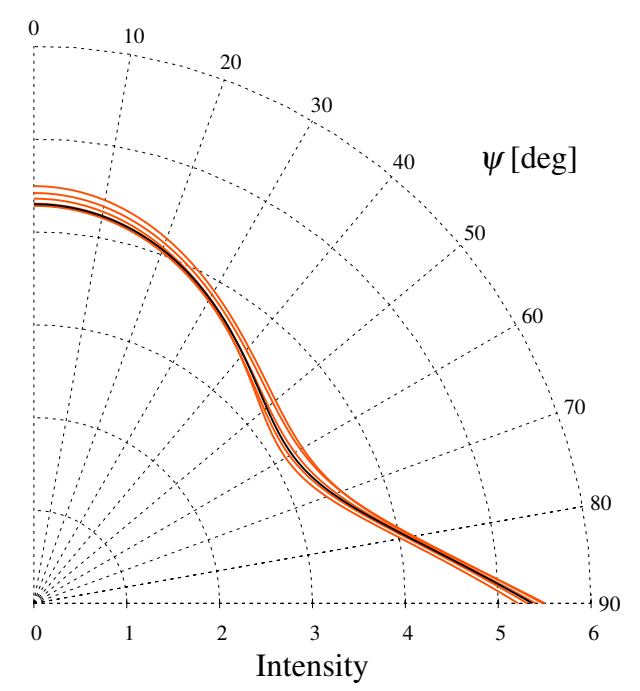

Figure 9: The initial upward angular intensity distribution function (thick solid line), and its best predictions at different scattering asymmetry parameter $h=0.0,0.1,0.2,0.3$, and $0.4 \mathrm{~km}$. The intensity scale is arbitrary. artificial lights in the atmosphere by a Monte-Carlo radiation transfer code. The use of Monte-Carlo simulation has turned out to be a good choice, since single or even double scattering is not suitable for the correct inversion of the system. Although our calculations are limited to a two parameter model of the atmosphere, elevation differences (topography) was ignored, we reach some firm conclusions:

- Measuring the sky luminance or radiance at a semicircular curve on the sky at two different distances from the light source provides enough information to get a rough estimate on the angular intensity distribution of the source and the parameters of the atmospheric model.

- The fitted model can be used to interpolate and extrapolate the sky luminance distribution at other locations than the points where the observations were taken. This makes a possible way to construct light pollution maps from limited number of ground based observations.

- Calculations with single scattering are not suitable for sky glow inversion. Even double scattering can underestimate the effect of the atmosphere, when the visibility is less than $20-30 \mathrm{~km}$.

We plan to extend our calculations for non-axial symmetric situations. The parallel, stereographic measurements can be taken to add the missing information. We also plan to perform real life tests of the method in the existing and planned dark sky places in Hungary, and later extend the survey for a wider area. Imaging photometry monitoring together with the model calculations of light pollution can be a well suited qualifying tool on the energy waste and on the nature of urban lighting.

Light scattering generally depends on wavelength of light. For public lighting white LEDs become more and more popular. It is of higher interest what kind of effects may be experienced in light pollution if the change from Na-lamps to LEDs in luminaires is performed. Photometric quantities related to night (scotopic) vision also play an important role, especially for darks sky parks and reserves. For example, our method makes it possible to predict the possible degradation of scotopic night sky quality based on recent imaging measurements, the models, and the spectra of the recent and planned light sources. But due to volume constraints this study will be the subject of another paper.

\section{Acknowledgements}

This work has been supported by the Hungarian OTKA grant K83790.

\section{References}

[1] Duriscoe DM, Luginbuhl CB, Moore CA. Measuring Night-Sky Brightness with a Wide-Field CCD Camera,. Publ. Ast. Soc. Pacific 2007;119:192. 


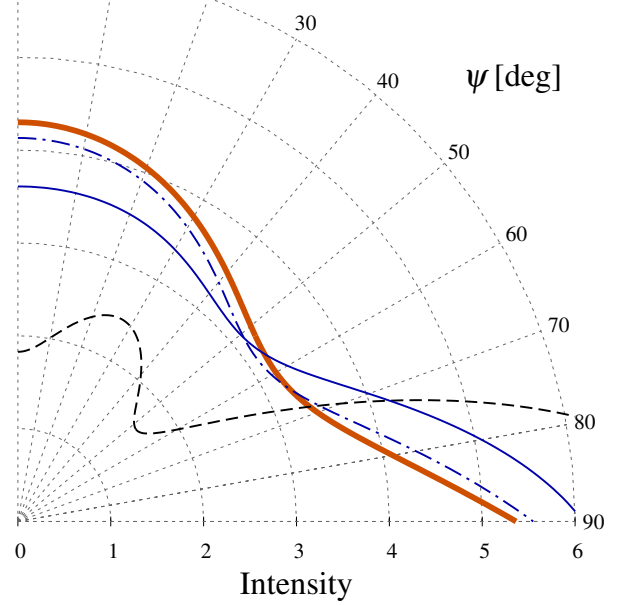

Figure 10: Effect of single (black dashed curve), double (blue solid curve), and triple (blue dashed-dotted curve) scattering on the fitted upward intensity distribution function. The intensity scale is arbitrary.

[2] Kolláth Z. Measuring and modelling light pollution at the Zselic Starry Sky Park. J. Phys.: Conf. Ser. 2010;218:012001.

[3] Cinzano P, Falchi H. The propagation of light pollution in the atmosphere. Monthly Notices of the Royal Astronomical Society 2012;427:3337-3357.

[4] Aubé M, Kocifaj M. Using two light-pollution models to investigate artificial sky radiances at Canary Islands observatories. Mon. Not. R. Astron. Soc. 2012;422:819-830.

[5] Spada F, Krol MC, Stammes P. McSCIA: application of the equivalence theorem in a Monte Carlo radiative transfer model for spherical shell atmospheres,. Atmospheric Chemistry and Physics Discussions 2006;6:4823

[6] Yusef-Zadeh F, Morris M, White RL. Bipolar reflection nebulae - Monte Carlo simulations. Astrophysical Journal 1984;278:186194.

[7] CIE. Colorimetric Observers, CIE S002. 1986.

[8] Garstang RH. Model for artificial night-sky illumination. Publication of the Astronomical Society of the Pacific 1986;98:364375.

[9] Kattawar GW. A three-parameter analytic phase function for multiple scattering calculations. J. Quant. Spectrosc. Radiat. Transfer 1975;15:839-849. 